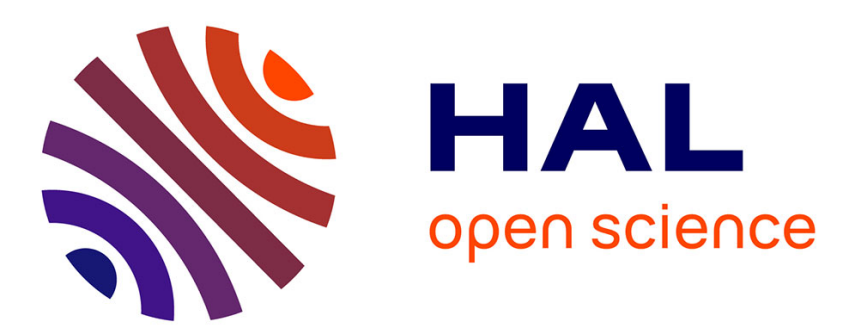

\title{
Practical Aspects of Super-Resolution Imaging and Segmentation of Macromolecular Complexes by dSTORM
}

\author{
Leonid Andronov, Jean-Luc Vonesch, Bruno Klaholz
}

\section{- To cite this version:}

Leonid Andronov, Jean-Luc Vonesch, Bruno Klaholz. Practical Aspects of Super-Resolution Imaging and Segmentation of Macromolecular Complexes by dSTORM. Multiprotein Complexes, 2247, Springer US, pp.271-286, 2021, Methods in Molecular Biology, 10.1007/978-1-0716-1126-5_15 . inserm-03376620

\section{HAL Id: inserm-03376620 https://www.hal.inserm.fr/inserm-03376620}

Submitted on 6 Dec 2021

HAL is a multi-disciplinary open access archive for the deposit and dissemination of scientific research documents, whether they are published or not. The documents may come from teaching and research institutions in France or abroad, or from public or private research centers.
L'archive ouverte pluridisciplinaire HAL, est destinée au dépôt et à la diffusion de documents scientifiques de niveau recherche, publiés ou non, émanant des établissements d'enseignement et de recherche français ou étrangers, des laboratoires publics ou privés. 


\title{
Practical aspects of super-resolution imaging and segmentation of macromolecular complexes by dSTORM
}

\author{
Leonid Andronov ${ }^{1,2,3,4,5}$, Jean-Luc Vonesch ${ }^{1,2,3,4,5}$ \& Bruno P. Klaholz $z^{1,2,3,4,5}$ \\ ${ }^{1}$ Centre for Integrative Biology (CBI), IGBMC, CNRS, Inserm, Université de Strasbourg, 1 rue \\ Laurent Fries, 67404 Illkirch, France. \\ ${ }^{2}$ Institute of Genetics and of Molecular and Cellular Biology (IGBMC), 1 rue Laurent Fries, \\ Illkirch, France. \\ ${ }^{3}$ Centre National de la Recherche Scientifique (CNRS), UMR 7104, Illkirch, France. \\ ${ }^{4}$ Institut National de la Santé et de la Recherche Médicale (Inserm), U964, Illkirch, France. \\ ${ }^{5}$ Université de Strasbourg, Illkirch, France.
}

\begin{abstract}
Super-resolution fluorescence microscopy allows imaging macromolecular complexes down to the nanoscopic scale and thus is a great tool to combine and integrate cellular imaging in the native cellular environment with structural analysis by $\mathrm{X}$-ray crystallography or highresolution cryo electron microscopy or tomography. Here we describe practical aspects of SMLM imaging by dSTORM, from the initial sample preparation using mounting media, antibodies and fluorescent markers, the experimental setup for data acquisition including multi-color colocalization and 3D data acquisition, and finally tips and clues on advanced data processing that includes image reconstruction and data segmentation using 2D or 3D clustering methods. This approach opens the path towards multi-resolution integration in cellular structural biology.
\end{abstract}




\section{Introduction}

Fluorescence microscopy is a key technique for the specific observation of proteins in their native cellular environment. Unfortunately, the resolution of fluorescence microscopy is limited by the wavelength of light to around $200-300 \mathrm{~nm}$, which limits the studies to the scale of cellular organelles. The recently emerged super-resolution fluorescence microscopy allows to overcome this limit and improves the resolution by about one order of magnitude, i.e. down to the nanoscopic scale of large macromolecular complexes. Super-resolution microscopy is therefore a powerful technique that bridges the gap between high-resolution crystallographic and cryo electron microscopy (cryo-EM) structures of proteins and their functions in the cellular context (1).

Among the super-resolution techniques, single-molecule localization microscopy (SMLM), which includes techniques such as (direct) stochastic optical reconstruction microscopy ((d)STORM) (2, 3), photoactivated localization microscopy (PALM) (4) and points accumulation for imaging in nanoscale topography (PAINT) (5), has proven to produce the highest resolution even using basic optical setups and common sample preparation protocols. SMLM relies on stochastic switching of fluorophores between a fluorescently active "on" and a fluorescently inactive "dark" state that can be controlled by the intensity of the excitation light and composition of the sample mounting medium. Using appropriate buffer and imaging conditions, many different fluorophores can be made suitable for SMLM, including organic fluorophores (6) and fluorescent proteins (7). SMLM not only provides super-resolution images, but also gives access to properties of individual molecules, allowing cluster analysis (8), segmentation (9), colocalization estimation (10), molecular counting (11) and probing of stoichiometry of macromolecular complexes (12).

In this chapter, we describe a common dSTORM experiment from sample preparation to image acquisition and data processing, which includes event list generation, image reconstruction and post-processing of SMLM data that can eventually be analyzed and segmented by 2D and 3D clustering methods. 


\section{Materials}

\subsection{Cell culture and immunolabelling}

In addition to your favorite adherent cell line, you need

1. Glass-bottom petri dishes with a diameter of $35 \mathrm{~mm}$ for cell culture (CELLView, Greiner BioOne)

3. Phosphate-buffered saline (PBS), 1 and 10 times concentrated (PBS 1x and 10x)

4. Formaldehyde $4 \%$

5. $0.1 \%$ Triton $\mathrm{X}-100$ in PBS (PBS/Tx)

6. Primary antibody

7. Secondary antibody coupled to compatible fluorophores (if primary antibody not directly labelled)

8. Bovine serum albumin (BSA)

9. Normal goat serum (NGS)

10. Fetal bovine serum (FBS)

Compatible fluorophores :

Alexa Fluor family (Thermo Fisher Scientific): Alexa 647 (the most commonly used fluorophore for SMLM), Alexa 555, Alexa 532, Alexa 488; Atto 488 (ATTO-TEC GmbH); Cy3B (13) and others (6).

\subsection{Mounting media: composition and choice}

Gloxy (6): Cysteamine hydrochloride $50 \mathrm{mM}$, glucose $10 \% \mathrm{w} / \mathrm{v}$, glucose oxidase $0.5 \mathrm{mg} / \mathrm{ml}$, catalase $40 \mu \mathrm{g} / \mathrm{ml}$ in TN buffer $(50 \mathrm{mM}$ Tris $(\mathrm{pH} 8.0)$ and $10 \mathrm{mM} \mathrm{NaCl})$.

We use the following stock solutions:

1. Cysteamine hydrochloride (Sigma-Aldrich $\mathrm{M} 6500,1 \mathrm{M}$ in $\mathrm{H}_{2} \mathrm{O}$, stored at $-20^{\circ} \mathrm{C}$ )

2. Glucose $400 \mathrm{~g} / 1$, stored at $-20^{\circ} \mathrm{C}$

3. Glucose Oxidase (Sigma-Aldrich $\mathrm{G} 2133,5 \mathrm{mg} / \mathrm{ml}$, stored at $4^{\circ} \mathrm{C}$ )

4. Catalase (Sigma-Aldrich C3515, $4 \mathrm{mg} / \mathrm{ml}$, stored at $4{ }^{\circ} \mathrm{C}$ ) 
Vectashield/TDE (14): Vectashield 20\% v/v, TDE $70 \% \mathrm{v} / \mathrm{v}$ in PBS.

1. Vectashield ${ }^{\circledR}$ Antifade Mounting Medium (Vectorlabs H-1000, stored at $+4^{\circ} \mathrm{C}$ )

2. 2,2'-Thiodiethanol (TDE, Sigma-Aldrich 166782)

Mix 20\% v/v Vectashield with 70\% v/v TDE and 10\% v/v PBS 10x. For mounting, incubate in solutions with increasing concentrations of TDE in PBS: 10\%, 25\%, 50\%, 10 min each. Then replace with the final solution, Vectashield/TDE.

OxEA (15): Cysteamine hydrochloride $50 \mathrm{mM}$, OxyFluor ${ }^{\mathrm{TM}} 3 \% \mathrm{v} / \mathrm{v}$, sodium DL-lactate 20\% v/v in PBS adjusted to $\mathrm{pH} 8-8.5$ with $1 \mathrm{M}$ aqueous $\mathrm{NaOH}$.

We use the following stock solutions:

1. Cysteamine hydrochloride (Sigma-Aldrich M6500, $1 \mathrm{M}$ in PBS, stored at $-20^{\circ} \mathrm{C}$ )

2. OxyFluor ${ }^{\mathrm{TM}}$ (Sigma-Aldrich SAE0059, stored at $-20^{\circ} \mathrm{C}$ ) (16)

3. Sodium DL-lactate (Sigma-Aldrich L1375, $60 \% \mathrm{w} / \mathrm{w}$ syrup, stored at $+4^{\circ} \mathrm{C}$ )

\subsection{Intrumentation}

The super-resolution experiments were performed on a Leica SR GSD system built on the base of the DMI6000 B inverted wide-field microscope, and consisting of the HCX PL APO 100x/1.47 Oil CORR TIRF PIFOC objective and 1.6x magnification lens that provide an equivalent pixel size of $100 \mathrm{~nm}$ on an EMCCD camera (Andor iXon3 DU-897U-CS0-\#BV); continuous wave fiber lasers (MPBC Inc., $488 \mathrm{~nm} 300 \mathrm{~mW}, 532 \mathrm{~nm} 1000 \mathrm{~mW}, 642 \mathrm{~nm} 500 \mathrm{~mW}$ ), a diode laser $(405 \mathrm{~nm} 30 \mathrm{~mW})$ and the suppressed motion (SuMo) sample stage. 


\section{Methods}

\subsection{Specimen preparation}

Procedure (adapted from a Leica protocol):

1. Seed cells in glass-bottom Petri dishes $\mathrm{d}=35 \mathrm{~mm}$ (CELLView, Greiner Bio-One) at a density of $\sim 100$ cells $/ \mathrm{mm}^{2}$.

2. Aspirate the cell culture medium.

3. Wash briefly $1 \mathrm{x}$ with PBS.

4. Fix with $4 \%$ formaldehyde in PBS for 20 min.

5. Permeabilize with PBS/Tx twice for $10 \mathrm{~min}$.

6. Incubate with primary antibody overnight at $4^{\circ} \mathrm{C}$ (see notes 1 and 2).

7. Wash $3 x$ with PBS/Tx over 2 hours each time.

8. Incubate with secondary antibody for 2 hours at RT.

9. Wash $3 x$ with PBS/Tx over 2 hours each time.

10. Postfix with $4 \%$ Formaldehyde in PBS for 10 min (see note 3).

11. Wash briefly $3 \mathrm{x}$ with PBS.

12. Store at $4^{\circ} \mathrm{C}$ in PBS until mounting.

13. Before imaging, replace PBS with an appropriate mounting medium.

The mounting medium for dSTORM (also known as the imaging buffer) has to be chosen based on following criteria:

1. Fluorophores used. Alexa-647 works best in Gloxy or Vectashield-containing media, while OxEA allows to use a wider range of dyes, e.g. for multi-color imaging. Vectashield-containing media are usually unsuitable for detection of light with wavelengths $\lesssim 550 \mathrm{~nm}$ and for excitation with wavelengths $\lesssim 500 \mathrm{~nm}$ due to autofluorescence.

2. Depth of the ROI inside the sample. A ROI close to the coverslip $(<200 \mathrm{~nm})$ can be imaged with total internal reflection fluorescent microscopy (TIRF) which allows to reduce background fluorescence. This needs a mounting medium with a refractive index significantly lower than that 
of glass. Therefore, usually water-based media are used $(\mathrm{n} \approx 1.33)$ : e.g. Gloxy or OxEA. These media, however, are less suitable for deeper (more than several $\mu \mathrm{m}$ from the coverslip) imaging because of spherical aberrations due to the refraction index mismatch. For such situations buffers with a refractive index, close to that of glass $(\mathrm{n} \approx 1.52)$, are better suited: e.g. Vectashield/TDE.Using highly inclined and laminated optical sheet (HiLo) illumination the sample is excited with a light beam under an angle smaller than the critical angle for the given coverglass/specimen interface, that allows to image deeper in the sample as compared with TIRF microscopy but allows to keep low background intensity (17). HiLo illumination can be used with any mounting medium.

3. Labelling method. Cysteamine- or $\beta$-mercaptoethanol-containing buffers may cleave antibodies which can decrease the specific labelling and increase background, while TDE-containing media destabilize phalloidin labelling (18).

4. Possibility to refresh the buffer. Gloxy buffer needs to be replaced each 2-4 hours, OxEA - each 6-12 hours, while Vectashield-based buffers do not need to be replaced.

\subsection{Basic SMLM experiment}

Workflow of acquisition-processing

A typical acquisition session is composed of the following steps.

1. Preview. It consists in the search of the region of interest (ROI) and focusing using conventional epifluorescence or TIRF/HiLo illumination with low laser power (1-5\% of maximal power). In the case of the TIRF/HiLo mode the best direction (azimuth) of laser illumination to a sample has to be chosen as well. The step is completed by capturing a wide-field image (Fig. 1A). It is important that the dSTORM experiment will be carried out under the same conditions ( $\mathrm{x}, \mathrm{y}, \mathrm{z}$ position, epi/TIRF, direction of laser illumination if TIRF/HiLo) to have a direct comparison with the classical (epifluorescence) microscopy image and avoid artifacts in the super-resolution image.

2. Pumping. Illumination of the sample with high intensity light in order to bring most fluorescent molecules into the dark state. At the very beginning, when all the molecules are in the on state, due to very strong excitation, the fluorescence is very intense because all the dyes emit at the same 
time. After a little time (depends on the kinetics of the fluorophore) with occupation of the dark state and depletion of the on state the intensity of fluorescence decreases because progressively less molecules stay in the on state until the number of shining molecules becomes less than one per diffraction-limited region. At this time one begins to see the light from each "on" molecule separately and it is the time to start the acquisition, the next step of the experiment.

3. Acquisition. With constant laser intensity, that may be as high as during pumping or lower, one gets a number of shining single-molecule events ("blinks") per frame with certain exposure time (Fig. 1B). This number is progressively dropping with photobleaching of the molecules, but it can be increased using "backpumping" (e.g. with a 405 nm-laser). With gradual increase of backpumping laser intensity, it is possible to keep the number of events per frame at a nearly constant value, until most of the molecules become bleached and the acquisition is finished.

4. Post-processing. Although real-time super-resolution image reconstruction is possible depending on installed software, a post-processing step is usually required to improve the result by optimizing the processing parameters. For example, to get rid of noise it is necessary to increase the threshold of single-molecule detection, if it was quite low during the experiment. And vice versa, if the threshold was pretty high, it is desirable to reduce it in order to get more events and obtain a better reconstructed image. The frames with undesirable artifacts (non-blinking areas, especially in the very first frames) should be truncated thus also improving the results.

\subsection{Experimental parameters of dSTORM data collection and processing}

There are numerous experimental parameters that can be changed by the user, summarized here.

1. Laser power, can be chosen differently for pumping, acquisition and backpumping. Strong excitation intensity during acquisition increases the speed of "blinking", reduces the number of "on" molecules and insures faster completion of the acquisition. However, too strong excitation can also lead to increase of the background brightness and reduction of the number of the localized molecules. This parameter has to be adjusted for each combination of fluorophore-imaging buffer. Backpumping intensity is normally set to zero for pumping and beginning of acquisition and is turned on with gradually increasing intensity after dropping the number of events per frame during acquisition. 
2. Frame exposure time. Ideally, it has to be equal to the average on-time of the fluorophores. If the exposure time is too short, molecules will appear on many consecutive frames, their signal to noise ratio (SNR) will be reduced. On the other side, if the exposure time is too long, the density of localizations per frame will be higher and the SNR can be also reduced because the background is acquired during the whole frame exposure time while the signal from the fluorophores is detected only during their on-time.

3. Illumination mode: epi-/HiLo/TIRF, azimuth of laser illumination (when available). Affects the penetration depth and the axial resolution. It is advised to make pumping in epi-illumination mode to send higher energy to the specimen and move to the dark state all the volume of the sample; this provides lower background during the acquisition.

4. EM camera gain for EMCCD detectors. Normally is set up to maximum, but to be diminished in case of saturation due to strong fluorescence and/or high exposure times (i.e. adjust and calibrate the dynamic range of the camera).

5. Threshold of single-molecular detection in photons/pixel. Affects the real-time reconstructed super-resolution image (if available); it can be changed in the post-processing step. Usually lower threshold increases the noise level, a high threshold reduces the number of events, so a compromise value should be found. It is convenient to follow which spots on the images are detected and adjust the threshold in a way that only the single-molecule spots are detected (Fig. 1C).

6. Pixel size for real-time reconstruction (Fig. 1D), if available. Commonly set to around $20 \mathrm{~nm}$, can be reduced for post-processing (e.g. 5 or $10 \mathrm{~nm}$ sampling).

\subsection{D SMLM experiment}

One of the easiest and most common ways for 3D SMLM imaging is realized by modification of the point spread function (PSF) of the microscope with an astigmatic aberration.

Calibration. The astigmatic PSF deformation as a function of the axial position of fluorophores should be calibrated by imaging fluorescent beads using several known axial positions of the objective. 
1. Install a sample with fluorescent beads with subdiffraction size in the microscope. Can be used, e.g. multi-color TetraSpeck ${ }^{\mathrm{TM}}$ Microspheres, 0.1 or $0.2 \mu \mathrm{m}$.

2. Put in place the cylindrical lens or activate astigmatism in the adaptive optics module.

3. Focus the microscope in a way that the images of the beads are closest to circularly symmetrical.

4. Adjust the imaging parameters (excitation intensity, exposure time and electron-multiplying gain of the camera) to get good SNR. It is preferable to increase first the excitation intensity in order to keep the exposure time minimal.

5. Image the beads as a Z-stack with a step of $50 \mathrm{~nm}$ around $+/-0.6-1 \mu \mathrm{m}$ of the focus point determined in step 3.

6. Repeat the procedure 1-5 for each spectral channel.

7. Focus the microscope on the beads in one of the spectral channels. Image the beads through all the channels in this Z-position. This is necessary to take into account axial chromatic aberration.

8. Fit all the acquired images with a software for single-molecule detection, which will be used for following experiments. Refer to the manual of the software.

Once the calibration is done, 3D SMLM experiments can be performed with the conventional SMLM procedure, with the difference that the maximal density of switched-on molecules on a frame should be reduced to avoid overlap of stretched PSF spots.

\subsection{SMLM data processing}

The first step in SMLM data processing is the determination of the localization of each individual fluorophore to provide a full event list from which a super-resolution image can be reconstructed. The single-molecule localization precision and therefore the best attainable resolution, can be determined by the following formula (19):

$$
\left\langle\left(\Delta x^{2}\right)\right\rangle=\frac{\sigma^{2}}{N}+\frac{a^{2}}{12 N}+\frac{8 \pi \sigma^{4} b^{2}}{a^{2} N^{2}}
$$

where $\Delta x$ is the standard deviation (SD) of the localization error, $\sigma$ is the SD of the PSF, $N$ is the number of detected photons, $a$ is the pixel size and $b$ is the SD of the background noise. For best 
localization precision it is therefore important to keep a high number of detected photons from individual fluorophores, low background noise, small PSF and a sufficiently small camera pixel size (usually around $100 \mathrm{~nm}$ ).

Acquired images have to be processed for detection of single molecules (could be done in real time during experiments depending on microscopy system). This will produce a list of localizations, from which a super-resolution image can be built or other information can be extracted using advanced data processing methods. Most dedicated super-resolution microscopes are supplied with a software for single-molecule detection, image reconstruction and basic post-processing of localization data. In practice and for advanced data processing, however, specialized software packages can be needed.

Single-molecule detection requires first of all a good choice of the image fitting method. The most popular methods are:

1. Center of mass assement - the fastest method, but produces good results only in conditions of low density of localizations and low homogeneous background. This is a good method for the initial processing of the data (e.g. during acquisition).

2. Least-square fitting - usually intermediate in speed group of methods, provides best results when the models for the PSF or for the noise are hard to determine. Some of these methods can be used for localization of overlapping fluorophores (20).

3. Maximum-likelihood estimation - usually the slowest family of algorithms, requires a model of the PSF and of the noise. Gives best results, especially for weak signals, provided that the PSF shape and the noise model are correctly set (21). Some of these methods can be used for localization of overlapping fluorophores (22).

Even though algorithms for single-molecule detection are often supplied by the manufacturer of the super-resolution system, the available tools are often not sufficient, especially for difficult imaging conditions, such as overlapping localizations, non-homogeneous background, weak signal etc. For such conditions external software packages, such as ThunderSTORM (23), B-recs (24), SimpleSTORM (25) and others (reviewed in (26)) can be recommended. 


\subsection{Super-resolution image reconstruction}

Unlike in conventional microscopy, the image in SMLM cannot be acquired in a direct optical way, but should be calculated from the list of localizations, obtained after fitting. Therefore, there are several methods to calculate an image from SMLM data:

1. Histogram mode. The gray value of a super-resolution pixel equals to the number of events detected within the pixel's area. The fluorescence intensity (number of photons) is not taken into account. The pixel size chosen by the user strongly affects the gray levels of the image: in case of too small pixel sizes the image would contain only "zeros" and "ones", i.e. become binarized. This mode is fast to calculate but it can produce noisy and pixilated images, especially for weak density of localizations.

2. "Gaussian" mode. Every localization is represented as a Gaussian function with the width equivalent to the localization precision of the fluorophore, taking into account the number of detected photons. This image is slower to calculate than the histogram image, but it provides a smooth representation and allows to decrease pixel size without risk of pixilation. However, this mode can reduce the resolution of the image (27).

3. Local density mode. The pixel's gray values are calculated to be proportional to the local density of fluorophores in the neighborhood of the pixel. The local density can be estimated, e.g. using Voronoi diagrams (9) or Delaunay triangulations (27). Even though this method is slower than the

others (but still reasonable as in the order of minutes), it provides smooth images with preserved resolution even for weak signals.

\subsection{Post-processing of localization data}

Post-processing of localization data includes methods such as correction of drift and chromatic aberrations that are essential for most of experiments, and advanced processing methods, such as clustering and colocalization analysis that are necessary for some experiments, depending on biological question and sample type or acquisition setup (e.g. multi-color imaging or 3D data acquisition etc.). 
For drift correction there are two most feasible possibilities: 1) correction using fiducial markers; 2) correction with cross-correlation. For the first approach, photostable fiducial markers, such as fluorescent beads, gold nanoparticles or quantum dots (28), need to be incorporated and fixed within the sample, usually on the surface of the coverslip. The second approach does not need fiducials and uses properties that are intrinsic to localization data and rely only on computing (29). The cross-correlation method works well for contrasted structures with well-defined shape but can be less efficient for diffuse structures. It can also be recommended for imaging far from the coverslip because the fiducials usually can be robustly immobilized only on a glass surface.

For multi-color imaging, chromatic aberrations of the objective can cause a shift between images, captured with different wavelengths. To correct for this, the aberrations first should be calibrated by imaging a multi-color fiducial marker (e.g. beads) with the spectral channel to be used for the experiments. The detected from the fiducials shift should be subtracted from the single-molecule coordinates (30).

If the on-time of fluorophores is higher than the frame exposure time, their images will appear on several consecutive frames. These localizations can be combined by searching localizations within a circle of a given radius (usually around $50 \mathrm{~nm}$ ) around every localization in following frames. Using this option, it is possible to increase the number of photons per localization and therefore the localization precision. An inconvenience is that localizations of different fluorophores can be combined by error if they appear on consecutive frame (this is less likely when the densities of switched-on fluorophores is low). In some cases it can be also useful to remove localizations that appear on too many consecutive frames, eliminating non-blinking regions that can produce artifacts.

Localization event lists can be conveniently analyzed with the help of histograms as a function of the number of photons per localization (Fig. 2) and number of localizations per frame (Fig. 3). Localizations with too low photon counts can be removed thus improving average localization precision. Frames with too high number of localizations that correspond to very dense localizations that may compromise single-molecule detection can be removed as well. An additional problem for data quantification can be relocalization events (fluorophores that appear more than once during the acquisition); these can be addressed by taking into account the blinking behavior of fluorophores (31). 


\subsection{SMLM data segmentation and cluster analysis, from 2D to 3D}

Advanced SMLM data processing techniques include co-localization and cluster analysis. While for classical microscopy data these are commonly done on images, analysis of SMLM images is less optimal, because the image is not the primary data type and moreover they are not built in a unique way (see section "Super-resolution image reconstruction"). To get most information from the data it is preferred to process directly the localization coordinates.

Cluster analysis or segmentation aims for the detection of accumulations (clusters) of fluorescently labelled objects, such as proteins or nucleic acids. These detected clusters should be then analyzed for their size, density distribution etc. Two types of cluster analysis can be distinguished: determination of global properties of data and detailed analysis of individual clusters. For the first part of the analysis the method of choice is Ripley's K- and L-functions $(32,33)$. It allows to, first, test whether or not the experimental distribution follows a given pattern, and, second, to find the characteristic size of clusters (8). A similar method was also developed for the estimation of colocalization between two experimental distributions (10), it provides a colocalization value for a region that reflects the global colocalization extent within this region.

For detailed cluster analysis, the Density-Based Spatial Clustering of Applications with Noise (DBSCAN) method (34) can be used. However, to get optimal results it needs optimization of its parameters that can introduce a bias in the results. Recently emerged Voronoi tessellation-based methods $(9,35-37)$, not only allow for an unambiguous local density estimation and visualization of SMLM data but also for testing of datasets for clustering via comparison of the Voronoi polygons built on the experimental data with that of the reference distribution of points and for automated unbiased segmentation without parametrization $(9,37)$.

Some of these processing aspects can be found in packages for microscope control, but in many cases external tools have to be used. There are several packages dedicated for processing of localization data, namely ThunderStorm (23) that includes post-processing aspects such as image reconstructions in several modes, filtering and combination of localizations, drift correction and estimation of colocalization. ViSP (38) is a convenient tool for 3D visualization and basic segmentation. Lama (39) provides tools for quality control, resolution estimation, cluster analysis with Ripley's functions, DBSCAN and Ordering Points To Identify the Clustering Structure (OPTICS) (40) algorithms, coordinate-based colocalization and estimation of stoichiometry of 
molecular complexes (12). MIiSR (41) is focused on colocalization estimation and cluster analysis using the Ripley's, DBSCAN and OPTICS methods. PALMsiever (42) allows for combination of localizations, drift correction, DBSCAN cluster analysis and different rendering possibilities. SharpViSu (29) offers comprehensive tools for correction of chromatic aberrations and drift, selection and filtering of localizations, different visualization modes, resolution estimation and, through ClusterViSu (9), cluster analysis with the Ripley's function method and Voronoi-based automated segmentation. Our latest development, 3DClusterViSu, allows to perform Voronoidiagram-based segmentation of 3D data (37). Clearly, if it can be done for a given experiment, 3D analysis becomes the method of choice nowadays as compared to $2 \mathrm{D}$ analysis because it avoids artefacts from superposed structures (see details in Suppl. Mat. in (37) and it provides direct 3D information about the molecular organization in the cell, thus also facilitating the integration with molecular and atomic structures and opening the path towards cellular structural biology (1).

\section{Acknowledgements}

We thank Yves Lutz from the Imaging Centre for discussions on the adaptation of a Leica protocol and his contribution during earlier published work. This work was supported by CNRS, Association pour la Recherche sur le Cancer (ARC), Institut National du Cancer (INCa), Ligue nationale contre le cancer (Ligue), Agence National pour la Recherche (ANR) and the USIAS research fellowship program of the University of Strasbourg. The super-resolution microscope setup was supported by the Alsace Region and by the French Infrastructure for Integrated Structural Biology (FRISBI; ANR-10-INSB-05-01) and Instruct-ERIC.

Correspondence and requests for materials should be addressed to B.P.K. (klaholz@igbmc.fr). 


\section{References}

1. Orlov I, Myasnikov AG, Andronov L, et al (2017) The integrative role of cryo electron microscopy in molecular and cellular structural biology. Biol Cell 109:81-93

2. Rust MJ, Bates M, and Zhuang X (2006) Stochastic optical reconstruction microscopy (STORM) provides sub-diffraction-limit image resolution. Nat Methods 3:793-795

3. Linde $\mathrm{S}$ van de, Löschberger A, Klein T, et al (2011) Direct stochastic optical reconstruction microscopy with standard fluorescent probes. Nat Protoc 6:991-1009

4. Betzig E, Patterson GH, Sougrat R, et al (2006) Imaging Intracellular Fluorescent Proteins at Nanometer Resolution. Science 313:1642-1645

5. Sharonov A and Hochstrasser RM (2006) Wide-field subdiffraction imaging by accumulated binding of diffusing probes. Proc Natl Acad Sci 103:18911-18916

6. Dempsey GT, Vaughan JC, Chen KH, et al (2011) Evaluation of fluorophores for optimal performance in localization-based super-resolution imaging. Nat Methods 8:1027-1036

7. Lippincott-Schwartz J and Patterson GH (2009) Photoactivatable fluorescent proteins for diffraction-limited and super-resolution imaging. Trends Cell Biol 19:555-565

8. Owen DM, Rentero C, Rossy J, et al (2010) PALM imaging and cluster analysis of protein heterogeneity at the cell surface. J Biophotonics 3:446-454

9. Andronov L, Orlov I, Lutz Y, et al (2016) ClusterViSu, a method for clustering of protein complexes by Voronoi tessellation in super-resolution microscopy. Sci Rep 6:24084

10. Malkusch S, Endesfelder U, Mondry J, et al (2012) Coordinate-based colocalization analysis of single-molecule localization microscopy data. Histochem Cell Biol 137:1-10

11. Lee S-H, Shin JY, Lee A, et al (2012) Counting single photoactivatable fluorescent molecules by photoactivated localization microscopy (PALM). Proc Natl Acad Sci 109:17436-17441

12. Fricke F, Beaudouin J, Eils R, et al (2015) One, two or three? Probing the stoichiometry of membrane proteins by single-molecule localization microscopy. Sci Rep 5:14072

13. Cooper M, Ebner A, Briggs M, et al (2004) Cy3B ${ }^{\mathrm{TM}}$ : Improving the Performance of Cyanine Dyes. J Fluoresc 14:145-150

14. Olivier N, Keller D, Rajan VS, et al (2013) Simple buffers for 3D STORM microscopy. Biomed Opt Express 4:885-899

15. Nahidiazar L, Agronskaia AV, Broertjes J, et al (2016) Optimizing Imaging Conditions for Demanding Multi-Color Super Resolution Localization Microscopy. PLOS ONE 11:e0158884

16. Adler H and Spady G (1997) THE USE OF MICROBIAL MEMBRANES TO ACHIEVE ANAEROBIOSIS. J Rapid Methods Autom Microbiol 5:1-12

17. Tokunaga M, Imamoto N, and Sakata-Sogawa K (2008) Highly inclined thin illumination enables clear single-molecule imaging in cells. Nat Methods 5:159-161

18. Staudt T, Lang MC, Medda R, et al (2007) 2,2'-thiodiethanol: a new water soluble mounting medium for high resolution optical microscopy. Microsc Res Tech 70:1-9

19. Thompson RE, Larson DR, and Webb WW (2002) Precise nanometer localization analysis for individual fluorescent probes. Biophys J 82:2775-2783

20. Holden SJ, Uphoff S, and Kapanidis AN (2011) DAOSTORM: an algorithm for highdensity super-resolution microscopy. Nat Methods 8:279-280

21. Abraham AV, Ram S, Chao J, et al (2009) Quantitative study of single molecule location estimation techniques. Opt Express 17:23352-23373 
22. Quan T, Zhu H, Liu X, et al (2011) High-density localization of active molecules using Structured Sparse Model and Bayesian Information Criterion. Opt Express 19:16963-16974

23. Ovesný M, Křížek P, Borkovec J, et al (2014) ThunderSTORM: a comprehensive ImageJ plug-in for PALM and STORM data analysis and super-resolution imaging. Bioinformatics 30:2389-2390

24. Rouault H (2013) Brecs: Reconstruction of dense localization microscopy datasets,

25. Köthe U, Herrmannsdörfer F, Kats I, et al (2014) SimpleSTORM: a fast, self-calibrating reconstruction algorithm for localization microscopy. Histochem Cell Biol 141:613-627

26. Sage D, Kirshner H, Pengo T, et al (2015) Quantitative evaluation of software packages for single-molecule localization microscopy. Nat Methods 12:717-724

27. Baddeley D, Cannell MB, and Soeller C (2010) Visualization of Localization Microscopy Data. Microsc Microanal 16:64-72

28. Glushonkov O, Réal E, Boutant E, et al (2018) Optimized protocol for combined PALMdSTORM imaging. Sci Rep 8:8749

29. Andronov L, Lutz Y, Vonesch J-L, et al (2016) SharpViSu: integrated analysis and segmentation of super-resolution microscopy data. Bioinformatics 32:2239-2241

30. Erdelyi M, Rees E, Metcalf D, et al (2013) Correcting chromatic offset in multicolor superresolution localization microscopy. Opt Express 21:10978-10988

31. Annibale P, Vanni S, Scarselli M, et al (2011) Quantitative Photo Activated Localization Microscopy: Unraveling the Effects of Photoblinking. PLOS ONE 6:e22678

32. Ripley BD (1976) The Second-Order Analysis of Stationary Point Processes. J Appl Probab 13:255-266

33. Nicovich PR, Owen DM, and Gaus K (2017) Turning single-molecule localization microscopy into a quantitative bioanalytical tool. Nat Protoc 12:453-460

34. Ester M, Kriegel H-P, Sander J, et al (1996) A density-based algorithm for discovering clusters in large spatial databases with noise, Presented at the

35. Voronoi, George (1908) Nouvelles applications des paramètres continus à la théorie des formes quadratiques. J Für Reine Angew Math 1908:97-102

36. Levet F, Hosy E, Kechkar A, et al (2015) SR-Tesseler: a method to segment and quantify localization-based super-resolution microscopy data. Nat Methods 12:1065-1071

37. Andronov L, Michalon J, Ouararhni K, et al (2018) 3DClusterViSu: 3D clustering analysis of super-resolution microscopy data by 3D Voronoi tessellations. Bioinformatics

38. Beheiry ME and Dahan M (2013) ViSP: representing single-particle localizations in three dimensions. Nat Methods 10:689-690

39. Malkusch S and Heilemann M (2016) Extracting quantitative information from singlemolecule super-resolution imaging data with LAMA - LocAlization Microscopy Analyzer. Sci Rep 6:34486

40. Ankerst M, Breunig MM, Kriegel H-P, et al (1999) OPTICS: Ordering Points to Identify the Clustering Structure, In: Proceedings of the 1999 ACM SIGMOD International Conference on Management of Data, pp. 49-60 ACM, New York, NY, USA

41. Caetano FA, Dirk BS, Tam JHK, et al (2015) MIiSR: Molecular Interactions in SuperResolution Imaging Enables the Analysis of Protein Interactions, Dynamics and Formation of Multi-protein Structures. PLOS Comput Biol 11:e1004634

42. Pengo T, Holden SJ, and Manley S (2015) PALMsiever: a tool to turn raw data into results for single-molecule localization microscopy. Bioinforma Oxf Engl 31:797-798 


\section{Notes}

Note 1. Absence of any blocking reagents and blocking step is possible with highly specific antibodies. If necessary, BSA $1 \%$ or a mixture of decomplemented NGS (5\%) and FCS (1\%) can be used before step 6 .

Note 2. To avoid aggregates all stock solutions of antibodies should be centrifuged at $16000 \mathrm{~g}$ for $5 \mathrm{~min}$. All working solutions can be filtered on $0.22 \mu \mathrm{m}$ filters.

Note 3. The purpose of the post-fixation of the immune complexes is two-fold: first, avoiding cleavage of disulfide bounds of the antibodies (destroying the immunoreactivity) in the mounting buffers that often contain reducing agents like cysteamine or $\beta$-mercaptoethanol. Moreover, cysteamine solutions are also weakly basic ( $\mathrm{pH} 8-8.5)$, weakening the bindings. Second, reducing the mobility of the antibodies on the antigens (off and on reactions) that may affect quality of superresolution images. 


\section{Figures}

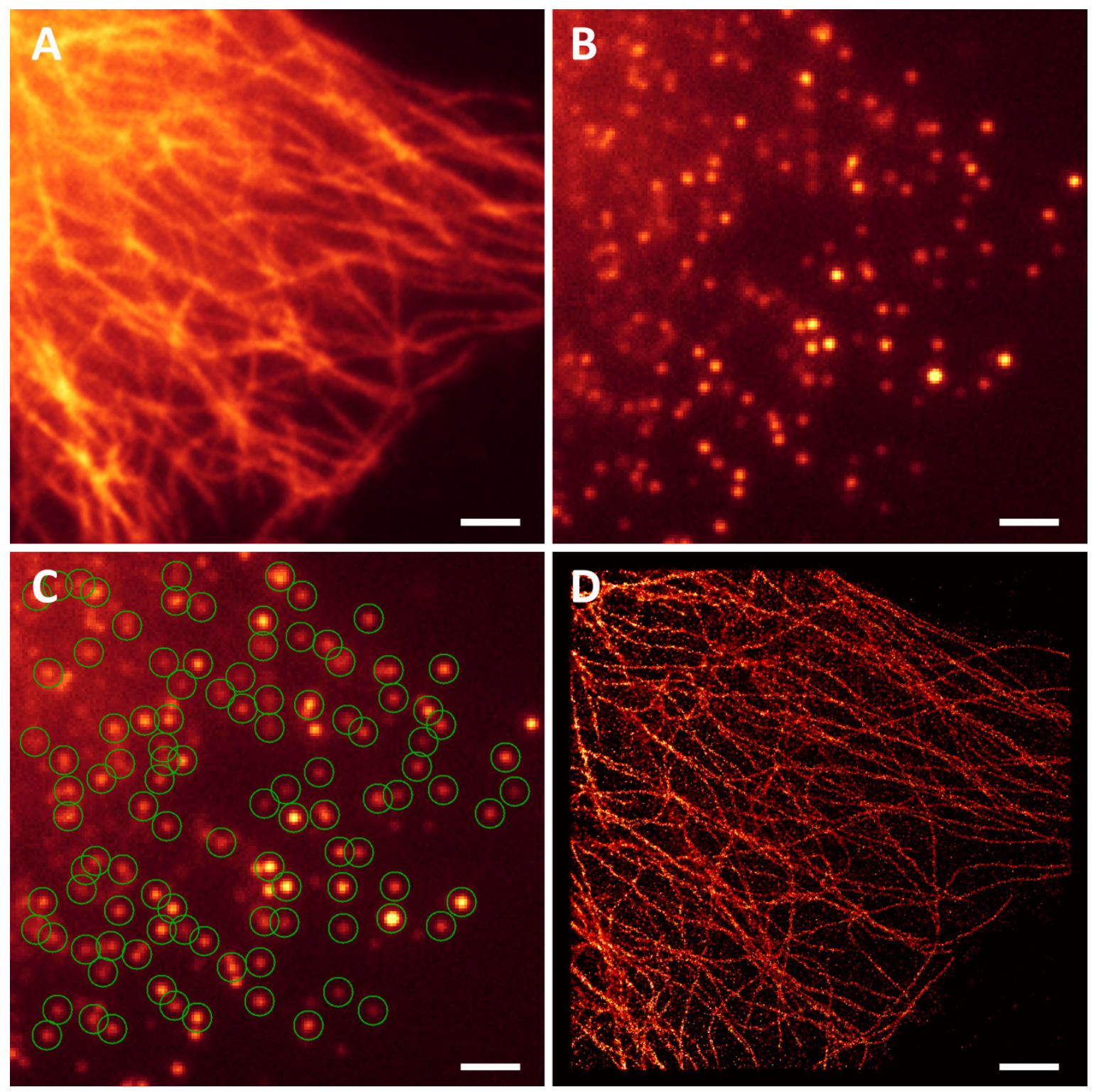

Figure 1. Example of a typical dSTORM data acquisition cycle (see methods). HeLa cells, $\beta$ tubulin marked by Alexa Fluor-647-conjugated secondary antibodies and mounted in the "Vectashield/TDE" medium. (A) Preview in HiLo mode. (B) One of the frames of the acquisition (exposure time is $50 \mathrm{~ms} /$ frame). (C) Circles indicate the spots that are selected by the Leica LAS AF software as single-molecule localizations. (D) Final reconstructed super-resolution image using 17622 frames with 1160875 localization events (collected here over $15 \mathrm{~min}$ ); the image is in the histogram mode with $20 \mathrm{~nm} / \mathrm{pixel}$, corrected for drift and rendered in SharpViSu (29). Scale bars, $2 \mu \mathrm{m}$. 


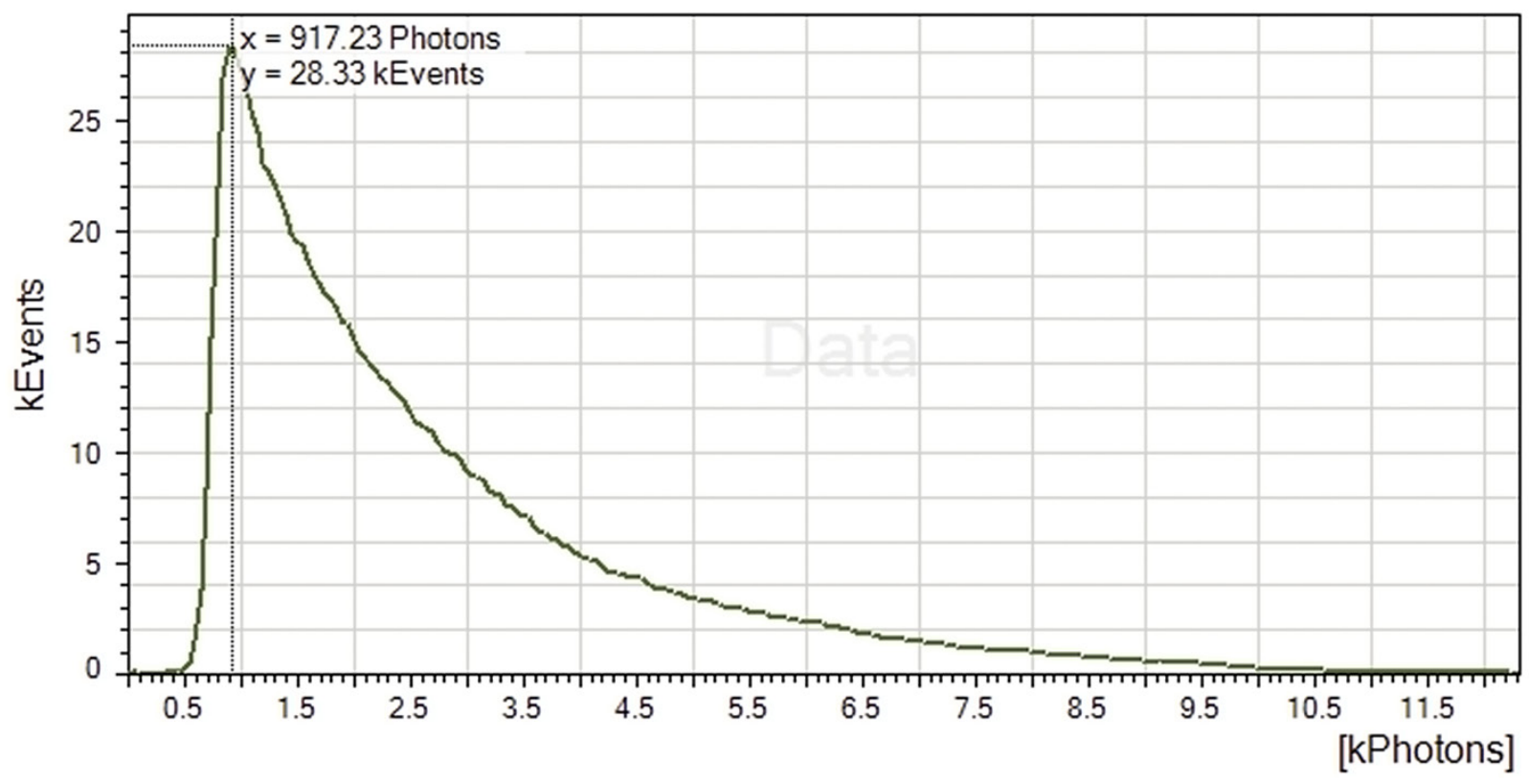

Figure 2. Typical histogram of the photon count of localizations of the Alexa Fluor-647 dye. The exposure time was $50 \mathrm{~ms} /$ frame, the detection threshold is 60 photons/pixel, 17622 frames were analyzed producing 1160875 events, the average photon count is 2675 photons/event. The analysis was done in the Leica LAS AF software. 


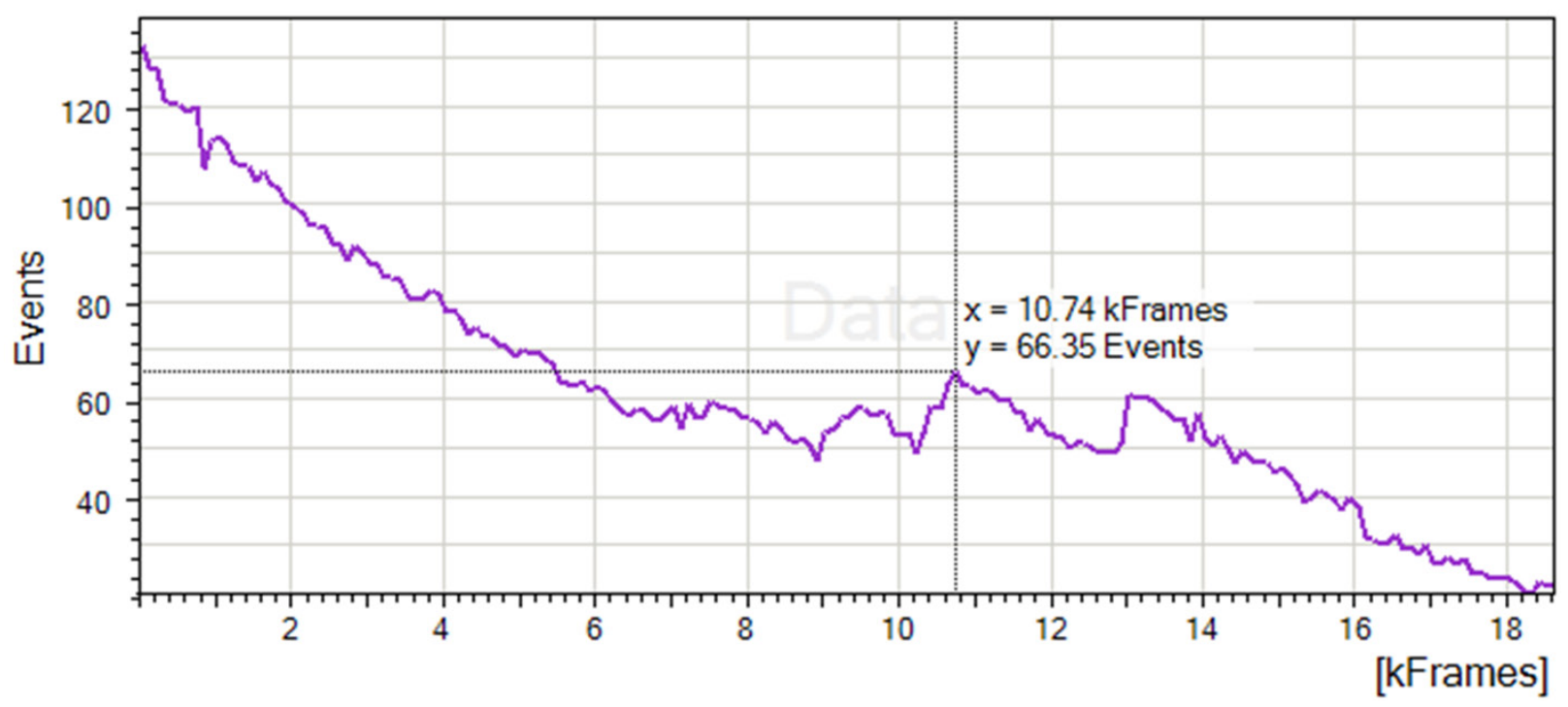

Figure 3. Typical histogram of the number of localizations per frame for the Alexa Fluor-647 dye. The curve is built using blocks of 100 frames each for smoothing reasons. Note increases in the number of localizations (middle part of the graph) due to an increase in intensity of the "backpumping" $405 \mathrm{~nm}$ laser. Exposure time is $50 \mathrm{~ms} / \mathrm{frame}$, detection threshold is 80 photons/pixel; 18620 frames with 1161303 events were analyzed. The analysis was done in the Leica LAS AF software. 